Eisenhydroxyd ab. Inwieweit sich diese anderweit vorteilhaft nutz. bar machen lassen, ist eine besondere Frage, die ich hier nar streifen will:

Beim Auflösen des Eisens in Salzsăure erbălt man ans der obigen Menge von $36,75 \mathrm{~kg}$ Eisen 1,3 kg Wasserstoffgas oder annähernd $15 \mathrm{cbm}$, die vielleicht als Beimischung zum Leuchtgase zur Erhöhung seiner Heizkraft verwendet werden könnten. Auch das bei der Chloridierung des Eisenchlorürs zu Eisenchlorid entstehende Manganchlorür ist jedenfalls von gewissem Werte. Fraglicher erscheint, ob sich das bei der Klärung des Wassers niederfallende und mit Schmutzstoffen aus dem Wasser beladene noch chlorhaltige Eisenbydroxyd, welches sich bei gröBeren Wasserwerken- in bedeutenden Mengen ansammeln würde, nutzbar verwerten ließe. Aber es will mir nicht unmöglich erscheinen, daß dasgelbe sich wieder verarbeiten ließe, vielleicht auch nach Reinigung von den Schmutzteilen wieder im Kreislauf des Prozesses in Eisenchlorid umgewandelt werden könnte.

Gelänge die Einführung solcher Nebenprozesse, so wăren dadurch die Kosten des Verfahrens auf ein Minimum zurückgeführt.

Aber auch im anderen Falle erscheint bei dem geringen Preise der Rohmaterialien, der Einfachheit der Herstellung und Anwendung der Eisenhydroxydlösung und ihrer unzweifelhaft großen Brauchbarkeit für die Wasserreinigung eine Prüfung des Verfahrens im Großbetriebe mit Rücksicht auf die oben (S. 21 Abs. 2) erwhbnten großen Vorteile sicher gerechtfertigt, zumal wo es sich um Leben und Gesundheit vieler Merschen handelt.

Mitteilung aus dem chemischen Untersuchungsamte der Stadt Aachen.

\title{
Eine neue Quecksilberlösung als Reagens auf Aldehyde, insbesondere Formaldehyd.
}

Von E. Feder.

(Eingegangen den 25. XI. 1906.)

Eine allgemeine Reaktion auf Aldehyde ergibt sich bekanntlich aus dem Verhalten derselben zu NeBler's Reagens; sie geben mit letzterem einen zunächst rotbraunen Niederschlag, der sich alsbald grau färbt. Weiter sind auch die Sachse'sche und die Knapp'sche Quecksilberlösang, die beide als Reagens aut Traubenzucker im Urin empfohlen sind, zur Untersuchung aut Aldehyde zu verwerten. Die Sachse'sche 
Lösuug enthält Quecksilberjodid-Jodkalium in alkalischer Lơsung, also dieselben Bestandteile, wie NeBler's Reagens, nur in anderer Konzentration. Die Quecksilberlösung nach Knapp wird aus Cyanquecksilber unter Zusatz von Alkali bereitet.

Die Empfindlichkeit von NeBler's Reagens gegenuber Aldebyden ist sehr groB. Der entstehende Niederschlag zeigt zunächst rotbranne Färbung. Nun ist aber auch der bekannte Niederschlag der NeBler'schen Lösung mit Ammoniaksalzen gelb bis gelbrot gefărbt; diese Eigenschaft des Reagens ist seiner Anwendung zur Untersuchung auf Aldehyde sicher nicht dienlich. Die Sachse'sche Quecksilberlosung gibt, wie schon ihre Zusammensetzung vermuten läßt, nicht nur mit Aldehyden eine Reaktion, sondern mit Ammonsalzen einen ahnlichen Niederschlag wie Neßler's Reagens. Die Knapp'sche Lösung reagiert allerdings nicht mit Ammonsalzen; doch ist sie andererseits lange nicht so empfindlich gegenilber Aldehyden. So gibt sie mit Spuren von Formaldehyd nar sehr allmählich eine Trübung unter Abscheidung von Quecksilber; Erwärmen beschleunigt den Vorgang.

Die Untersuchung auf Formalin wird im Laboratorium des Nahrungsmittel-Chemikers wohl zameist mit ammoniakalischer Silberlösung ausgeführt, bedingt dann jedoch eine durch mohrere Stunden fortgesetzte Beobachtung; bis zur Bildung einer 'Trübung dauert es bei geringen Mengen Formalin oft recht lange. Es ist da wohl von Wert, eine Reaktionsflüssigkeit zu besitzen, die mit Spuren Form. aldehyd in kürzester Zeit ein einwandfreies Resultat liefert.

Wenn man Quecksilberchloridlösung mit Natronlange versetzt und dann Natriumthiosulfatlösung hinzufügt, so erhält man eine klare Lösung, welche Quecksilberthiosulfat bezw. ein Doppelsalz mit Natriumthiosulfat enthält; man kanu sie herstellen, indem man zu einer $2 \%$ igen Quecksilberchloridlösung das gleiche Volum einer Lösung von $10 \mathrm{~g}$ Natriumthiosultat nnd $8 \mathrm{~g}$ Aetznatron in $100 \mathrm{ccm}$ Wasser fugt. Dieselbe gibt mit kleinsten Mengen Formaldehyd angenblicklich eine sich allmählich verstärkende Abscheidung ron metallischem Quecksilber. Mit Ammonsalzen reagiert die Flusssigkeit absolut aicht. Jedoch hat sie immerhin den Nachteil, daß relativ schnell eine Zersetzung derselben anter Trtbung stattfindet.

Wendet man anstatt des Natriumthiosulfates Natriumsulfit an, so resultiert eine weit stabilere Lösung, die ein vőllig wasserklares Aussehen aufweist. Sie gibt allerdings mit wenig Ammoniaksalz einen Niederschlag; jedoch ist sie einmal nicht so empfindlich wie NeBler's Reagens, sodann ist der entstehende Niederschlag weiß gefärbt und mit dem durch Aldehyde erzielten grauen Niederschlag von metallischem Quecksilber gar nicht zu verwechseln. 
Die Fllissigkeit wurde anf folgende Weise bereitet. Eine Lösung von $20 \mathrm{~g}$ Quecksilberchlorid in Wasser, zn einem Liter aufgefüllt, und andererseits eine solche von $100 \mathrm{~g}$ Natriumsulfit und $80 \mathrm{~g}$ Aetznatron, ebenfalls zu einem Liter anfgefüllt, wurden getrennt aufbewahrt. Bei jedesmaligem Gebrauch wurden dann gleiche Volumina beider Lösungen gemischt, and zwar wurde die alkalische Sulfitlösung unter Umschwenken schnell zu der Quecksilbersalzlosung hinzugefügt. Es resultierte dann, wie bereits erwäbnt, eine vollständig farblose, klare Lösung.

Dieselbe erwies sich als ein sebr empfindliches Reagens auf Aldehyde, besonders Formaldehyd. Einigermaßen beträchtliche Mengen des letzteren rufen augenblicklich eine Abscheidung von metallischem Quecksilber hervor; 0,2 mg Formaldehyd verursachte schon nach wenigen Sekunden (in $10 \mathrm{ccm}$ Reagens) eine deutliche, sich noch verstärkende Trübung. Sogar die minimale. Menge, von $0,05 \mathrm{mg}$ rief nach 1-2 Minuten noch eine recht deatliche Reaktion hervor. $50 \mathrm{~g}$ Schmalz, das mit sehr wenig Hexamethylentetramin versetzt war, warde mit $25 \mathrm{ccm}$ Wasser in einem Destillationskolben gebracht und nach Zusatz von etwas Salzsăure destilliert; die ersten $5-10 \mathrm{ccm}$ des Destillates ergaben mit dem Reagens eine augenblickliche Abscheidung von $\mathrm{Hg}$.

Der quantitative Verlauf der Reaktion wurde noch durch folgenden Versuch bewiesen. Der Gehalt einer Formalinlösung an Formaldehyd war nach G. Romijn za $0,174 \%$ ermittelt worden. Bei der geschilderten Reaktion reduziert ein Molekül $\mathrm{HC}_{\mathrm{H}} \mathrm{O}$ ein $\mathrm{Molekül} \mathrm{HgCl}_{2}$ zu metallischem $\mathrm{Hg} ; 200 \mathrm{mg} \mathrm{Hg}$ entsprechen also $30 \mathrm{mg} \mathrm{HC} \gtrless_{\mathrm{H}}$.

Zu $50 \mathrm{ccm}$ des Reagens wurde eine abgemessene Menge der obigen Formalinløsung mit einem Gehalt von 0,174\% Formaldehyd gefügt. Die Mischung wurde dann (einige Stunden) stehen gelassen, bis sie vollständig abgesetzt hatte. Nunmehr wurde das metallische Quecksilber im Allihn'schen Rohr gesammelt, mit $40-50^{\circ}$ warmem Wasser gut ausgewaschen, dann mit Alkohol und Aether nachgewaschen; sodann wurde mit der Luftpumpe ein mäßiger Strom von Luft durchgesaugt, welche in einer Waschflasche mit konzentrierter Schwefelsäure getrocknet war. Das auf die Weise gesammelte Quecksilber wurde sodann gewogen. Es wurden folgende Resultate erhalten ${ }^{1}$ ):

1) Von Interesse ist wohl die Bemerkang, dab bei einem Versuch, ob sich nach dem geschilderten Verfabren, auch umgekehrt, das Quecksilber quantitativ ausscheide, folgende Ergebnisse erhalten warden:

Gefunden 670,$7 ; 535,1 ; 266,6 ; 267,7 \mathrm{mg}$ metallisches $\mathrm{Hg}$ statt 676,$8 ; 541,8 ; 270,9 ; 270,9$

Dabei wurden zur Lösung der betr. Quecksilberchloridmenge in $25 \mathrm{ccm}$ Wasser $25 \mathrm{ccm}$ der beschriebenen alkalischen Sulfitlösung gesetzt und dann Formalin in Ueberschuß zugefügt. 
Gefanden 57,1;114,3 mg metall. Hg bezügl. entspr. 8,6;17,1 mg Formaldehyd statt 58,$0 ; 116,0$ n $n$ n statt 8,$7 ; 17,4$, n

Schließlich will ich noch bemerken, daß die beschriebene alkalische Quecksilberlösung sich, wie gegen andere Aldehyde, auch naturgemäß gegen Tranbenzucker verhält; bereits in der Kalte tritt nach kurzer Zeit eine kräftige Reduktion ein, die durch Erwärmen bedeutend beschleunigt werden kann. Doch kann das Reagens in der vorliegenden Konzentration zur Untersuchung von Harn auf Traubenzncker keine Verwendung finden, da dasselbe auch durch zuckerfreien Harn bereits redaziert wird.

Arbeiten aus dem chemischen Institut der tierärztlichen Hochschule zu Dresden. Mitgeteilt von H. Kunz-Krause.

\section{Ueber einige Cyklogallipharate und tuber das Verhalten der Cyklogallipharsäure zu Ferrichlorid.}

Von Hermann Kunz-Krause und Rudolf Richter.

(Eingegangen den 3. XII. 1906.)

Von Metallverbindungen der Cyklogallipharsäure sind in der vorhergehenden Mitteilung ${ }^{1}$ ) von dem einen von uns und Paul Schelle das Calcinm•, Silber- und Ferricyklogallipharat ${ }^{2}$ ) beschrieben worden. Aus der Ontersuchung zunăchst der beiden erstgenaunten Salze, in Verbindung mit den durch Titration der Cyklogallipharsh̆ure erhaltenen Werten ${ }^{8}$ ) ergab sich, daß in der Cyklogallipharsäure: $\mathrm{C}_{81} \mathrm{H}_{80} \mathrm{O}_{8}$ eine einbasische Monokarbonsaure: $\mathrm{C}_{20} \mathrm{H}_{34}<_{\mathrm{COOH}}^{\mathrm{OH}}$ vorliegt, und daß somit deren Salze nach der allgemeinen Formel: $\mathrm{C}_{20} \mathrm{H}_{84}<\mathrm{COOOM}$ zasammengesetzt sind. Die seitdem von uns, zugleich zum Zweck der Vervollständignng der allgemeinen Kenntnis der Salzverbindungen der Cyklogallipharsäure, ausgeführte Untersuchung einer Anzahl weiterer, im folgenden beschriebener Cyklogallipharate hat zur Bestätigung dieser ersten Ergebnisse gefübrt.

1) Dieses Archiv 242 (1904), S. 257.

9) Ebenda, SS. 258, 260, 261.

s) Ebenda, S. 261. 